\title{
Oclusão Percutânea de Fístula Aorto-Coronária Venosa Adquirida Após Revascularização Miocárdica: Experiência Inicial com Plug Vascular Autoexpansível de Nitinol
}

\author{
Manuel Pereira Marques Gomes Jr., Ricardo Peressoni Faraco, Manfredo Kenji Naritomi, \\ Elisabeth Tomoko Takitani, José Victor Kairiyama, Cláudia Maria Rodrigues Alves
}

\section{RESUMO}

Enxertos aorto-coronários anastomosados inapropriadamente no sistema venoso representam complicação infrequente, porém potencialmente grave, das cirurgias de revascularização miocárdica. Técnicas cirúrgicas e dispositivos percutâneos diversos têm sido descritos para a correção definitiva dessas fístulas. Relatamos aqui dois casos de fístulas aorto-coronárias iatrogênicas submetidas à oclusão percutânea com sucesso, utilizando-se dispositivo autoexpansível de nitinol (plug vascular).

DESCRITORES: Fístula arteriovenosa. Revascularização miocárdica. Doença iatrogênica. Intervenção coronária percutânea.

$\mathrm{F}$ ístulas coronárias são condições raras, congênitas ou adquiridas, que podem determinar insuficiência cardíaca de alto débito, hipertensão arterial pulmonar ou isquemia miocárdica, secundárias ao shunt esquerda-direita ou ao fenômeno do "roubo de fluxo" coronário. ${ }^{1}$

Enquanto um período de observação inicial em pacientes assintomáticos tem sido a regra, a presença de sintomas ou o aumento de tamanho progressivo dessas conexões anômalas determinam a necessidade de intervenção - percutânea ou cirúrgica. ${ }^{2-4}$

Relatamos aqui a experiência inicial de embolização percutânea de fístulas arteriovenosas (FAV) adquiridas após revascularização miocárdica cirúrgica, utilizando-se

\section{ABSTRACT}

Percutaneous Occlusion of Acquired Aorto-Coronary Venous Fistula After Coronary Artery Bypass Graft Surgery: Initial Experience Using the Self-Expandable Nitinol Vascular Plug

Inadequate anastomosis of aorto-coronary grafts to the coronary venous system represent an infrequent but potentially serious complication of coronary artery bypass surgery. Different surgical techniques and percutaneous devices have been described for the permanent correction of these fistulas. We report two cases of iatrogenic aorto-coronary fistulas successfully treated with percutaneous occlusion using the self-expandable nitinol device (vascular plug).

DESCRIPTORS: Arteriovenous fistula. Myocardial revascularization. latrogenic disease. Percutaneous coronary intervention.

um oclusor vascular autoexpansível de nitinol, em duas pacientes que se apresentavam com persistência de sintomas anginosos após revascularização miocárdica completa e tratamento medicamentoso otimizado.

\section{RELATO DOS CASOS}

\section{Caso 1}

Paciente do sexo feminino, 65 anos, com antecedentes de diabetes mellitus tipo 2, hipertensão arterial sistêmica e doença coronária crônica (revascularização miocárdica cirúrgica realizada em 2011), admitida em abril de 2013 devido à síndrome coronariana aguda de risco intermediário (escore GRACE: 96). Eletrocardiogramas seriados evidenciaram ritmo sinusal regular e au- 
sência de alterações compatíveis com isquemia aguda; os marcadores de necrose miocárdica encontravam-se negativos. Ecocardiografia transtorácica bidimensional subsequente demonstrou dilatação discreta de átrio direito e moderada em esquerdo. O ventrículo esquerdo apresentava pequena dilatação e função sistólica global com redução discreta devido à hipocinesia septal anterior (segmento médio-basal). A fração de ejeção (Simpson) foi de 0,53 e a pressão sistólica em artéria pulmonar de $24 \mathrm{mmHg}$.

A angiotomografia de coronárias evidenciou um enxerto de veia safena dilatado e conectado à veia cardíaca magna, com importante dilatação do seio coronário (Figura 1A).

A cinecoronariografia confirmou a presença da conexão do enxerto de safena com a veia cardíaca magna, com evidência adicional de lesão obstrutiva segmentar e grave no terço médio da artéria circunflexa não revascularizada (Figura 1B). Optou-se por intervenção coronária percutânea (ICP) da artéria circunflexa e programação ambulatorial para oclusão da FAV. Foi realizado, com sucesso, implante de stent com eluição de everolimus Xience Prime ${ }^{\circledR}$ (Abbott Vascular, Santa Clara, Estados Unidos) de 2,75 x 33 mm.

Quinze dias após a alta hospitalar, a paciente apresentou novo episódio de angina prolongada em repouso, de características similares ao evento prévio, suscitando nova admissão em unidade coronária. Nova cinecoronariografia evidenciou stent pérvio e, sequencialmente, procedeu-se à oclusão percutânea da FAV. Foram realizadas anticoagulação com heparina não fracionada $100 \mathrm{U} / \mathrm{kg}$ e cateterização seletiva do enxerto com cateter-guia Amplatz Right-2 de 7 F. Devido à tortuosidade proximal, optou-se por posicionar dois fios-guia 0,014" CholCE ${ }^{\circledR}$ PT Extra Support Guide Wire (Boston Scientific Corporation, Natick, Estados Unidos) distalmente, propiciando entubação profunda do cateter-guia (Figura 2A).

Sob controle fluoroscópico, foi avançado o plug vascular Amplatzer ${ }^{\circledR}$ Plug Vascular II 8,0 × 7,0 mm (St. Jude Medical, Minnetonka, Estados Unidos) até o segmento médio do enxerto, sendo recuados os fios-guia e, então, liberado o dispositivo. Angiografia de controle demonstrou a oclusão total do enxerto 5 minutos após a liberação da prótese (Figura 2B).

\section{Caso 2}

Paciente do sexo feminino, de 45 anos, com histórico de hipertensão arterial sistêmica, dislipidemia e doença coronária crônica. Submetida à ICP com implante de stents farmacológicos em tronco de coronária esquerda e artérias descendente anterior e circunflexa, além de duas revascularizações cirúrgicas (a última em outubro de 2012), com quadro atual de dor torácica atípica e dispneia aos esforços.

Durante investigação diagnóstica, realizou angiotomografia de coronárias, que caracterizou enxerto em Y de artéria radial para artéria descendente anterior e de veia safena com anastomose distal em veia cardíaca (Figura 3A).

A angiografia coronária demonstrou stents posicionados no tronco da coronária esquerda e nas porções proximais da artéria descendente anterior e circunflexa, com reestenose focal intra-stent grave no óstio de artéria circunflexa, confirmando a presença de FAV descrita na angiotomografia (Figura 3B). Foi efetuada a oclusão percutânea da FAV no mesmo
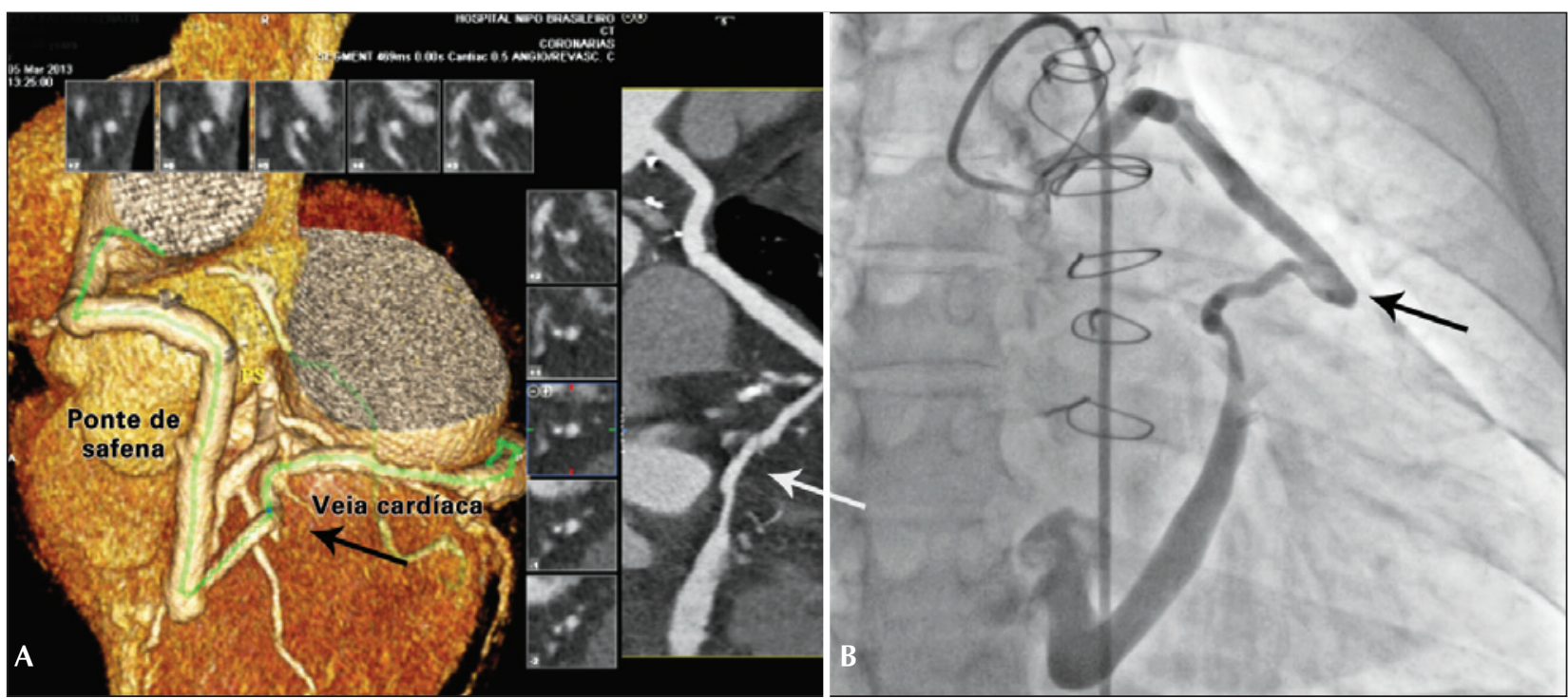

Figura 1. Caso 1: angiotomografia demonstrando enxerto de ponte de safena pérvio e dilatado, conectado à veia cardíaca magna (seta) em projeção oblíqua anterior esquerda (A) e angiografia do mesmo enxerto em oblíqua anterior direita (B). 

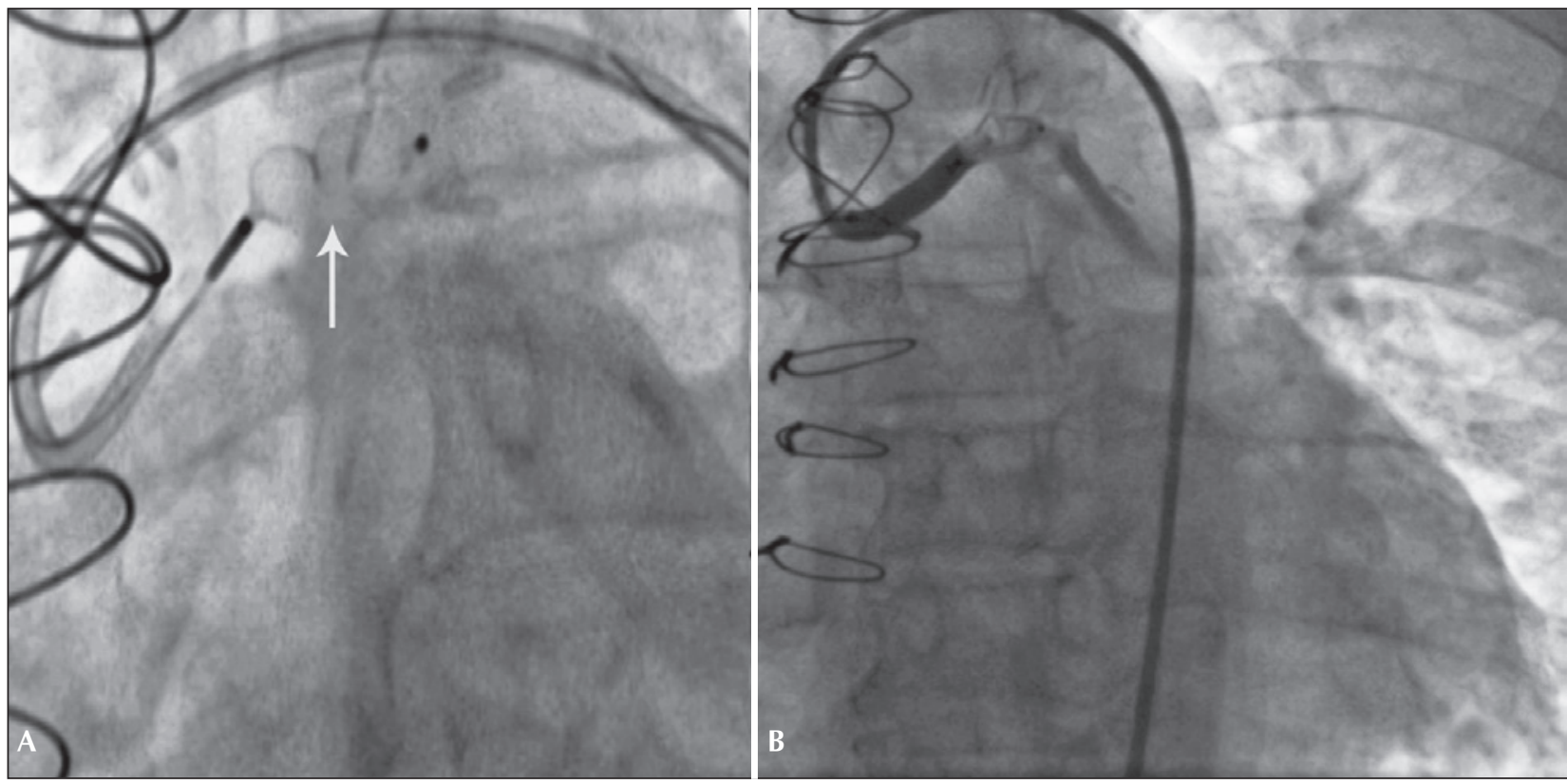

Figura 2. Caso 1: posicionamento do plug vascular autoexpansível (seta), imediatamente antes da liberação (A). Ausência de fluxo anterógrado no enxerto 5 minutos após a liberação do dispositivo (B).

procedimento. A cateterização seletiva do enxerto foi obtida com cateter-guia Amplatz Left-3 $6 \mathrm{~F}$, sob heparinização plena, utilizando-se fio-guia de 0,014" extrassuporte para intubação profunda. O dispositivo selecionado foi o Amplatzer ${ }^{\circledR}$ Plug Vascular II $10 \mathrm{x}$ $7,0 \mathrm{~mm}$ (Figura 3C) posicionado em ponte de safena, a montante da emergência do $\mathrm{Y}$ da artéria radial e liberado após a retirada de fio-guia. Observada discreta lentificação de fluxo anterógrado no enxerto de safena, com manutenção de sua patência.

Após 30 dias de evolução, a paciente foi admitida ao laboratório de intervenção para tratamento de reestenose intra-stent em artéria circunflexa e reavaliação do enxerto tratado. Submetida à ICP com o balão com eluição de paclitaxel Pantera Lux $^{\circledR} 3,0$ x 15 mm (Biotronik, Berlim, Alemanha) com sucesso. Angiografia de controle demonstrou oclusão completa em anastomose de ponte safena com a veia cardíaca, com manutenção do fluxo na anastomose em $\mathrm{Y}$ para descendente anterior (Figura 3D).

\section{DISCUSSÃO}

Fístulas coronárias adquiridas, decorrentes da inserção inadvertida de enxertos aorto-coronários (ou de artéria torácica interna in situ) em veias cardíacas, representam complicação rara e potencialmente grave das cirurgias de revascularização miocárdica. ${ }^{3}$

Em revisão recente, Gardner et al. ${ }^{4}$ reportaram apenas 36 casos publicados, nos quais a maioria das FAV envolvia o território da artéria descendente anterior $(66 \%)$ e de ramos diagonais (11\%).
Distorções na anatomia ventricular (fibrose e aderências pericárdicas secundárias a infarto, pericardite ou revascularização prévios), presença de trajeto intramiocárdico, gordura epicárdica sobrejacente e colapso vascular secundário à solução cardioplégica e ao clampeamento da aorta podem dificultar a identificação do vaso alvo e predispor à anastomose distal no sistema venoso. ${ }^{4}$

Sopro sistólico contínuo de alta frequência, insuficiência cardíaca sem causa definida e angina persistente após revascularização miocárdica devem suscitar a possibilidade de FAV iatrogênica. Os sintomas podem se manifestar desde o pós-operatório imediato até vários anos após a intervenção. ${ }^{2,5}$

A história natural dessas FAV depende da magnitude do shunt esquerda-direita desenvolvido. De modo similar às fístulas congênitas, falência cardíaca de alto débito, eventos trombóticos, dilatação progressiva com ruptura e hemopericárdio, hipertensão arterial pulmonar, arritmias atriais e ventriculares, e endarterite infecciosa são possíveis complicações. ${ }^{3,4}$ Alguns pacientes, todavia, permanecem assintomáticos e, eventualmente, apresentam oclusão espontânea da FAV. ${ }^{6}$

Isquemia miocárdica manifesta por positividade de provas funcionais ou como angina persistente tem como mecanismos principais a limitação de fluxo no território não revascularizado e o fenômeno de roubo de fluxo coronário (mais evidente em enxertos sequenciais). ${ }^{1,5} \mathrm{~A}$ ausência de perfusão miocárdica retrógrada, a partir do seio coronário arterializado (na ausência 


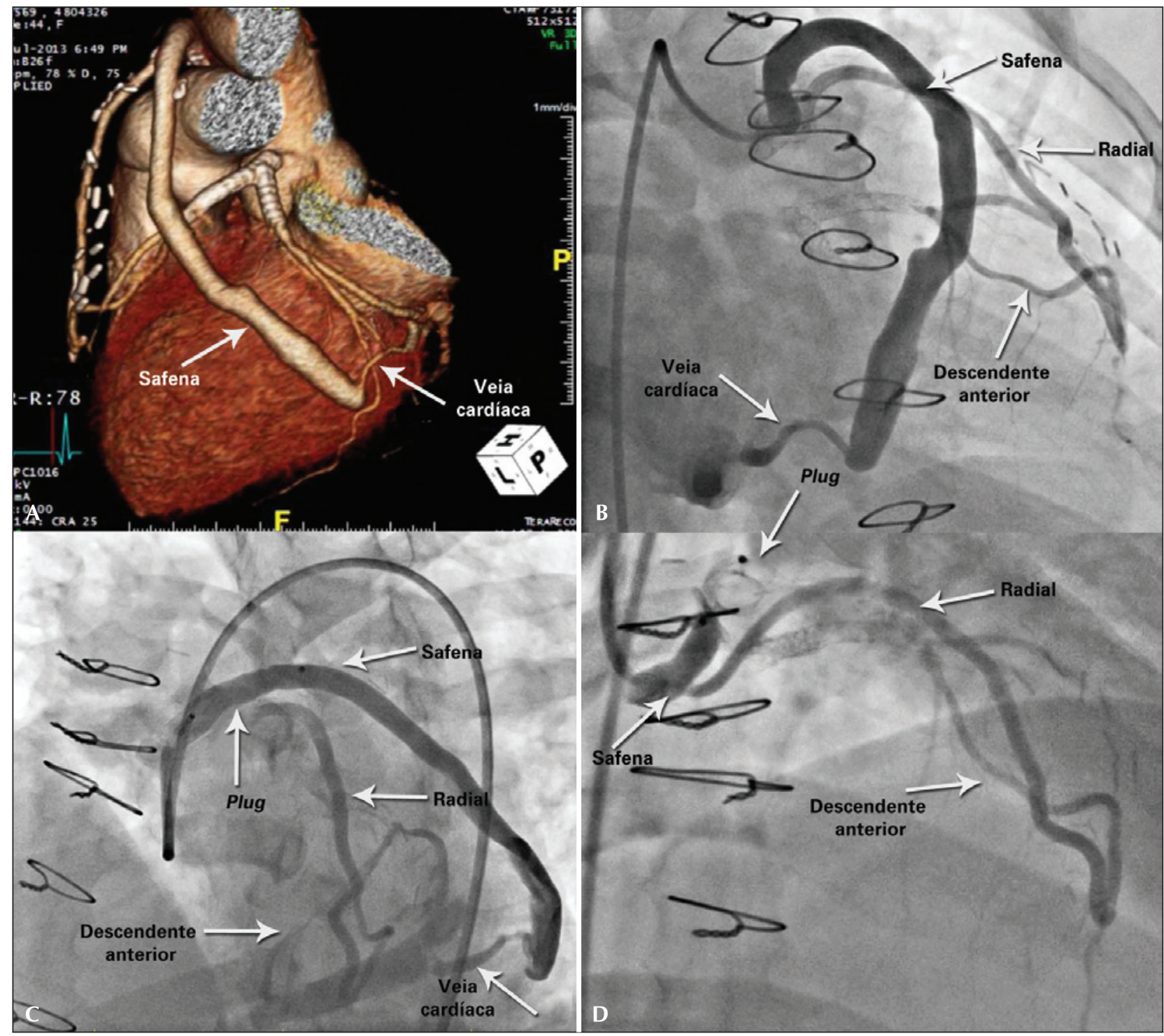

Figura 3. Caso 2: angiotomografia demonstrando anastomose safena-veia cardíaca (A). Angiografia demonstrando os dois ramos do enxerto em Y radial-descendente anterior e safena-veia cardíaca (B). Resultado imediato com amplo fluxo após liberação do plug, que pode ser visto pelas marcações proximal e distal (C). Resultado após 30 dias de procedimento, demonstrando o plug mais acomodado e oclusão do ramo para veia cardíaca, além de permeabilidade do Y para artéria descendente anterior (D).

de ligadura proximal da veia), pode potencializar a isquemia miocárdica nesses casos. $^{7}$

A cateterização cardíaca esquerda (angiografia coronária e manometrias) permanece como o padrão-ouro para a avaliação da anatomia, repercussão hemodinâmica e planejamento terapêutico das FAV. A angiotomografia e a ressonância cardíaca têm se mostrado de grande valia, proporcionando informações adicionais quanto ao trajeto, ao sítio de drenagem e à relação das FAV com outras estruturas.

Não existe consenso quanto à modalidade inicial de intervenção no grupo de pacientes sintomáticos 


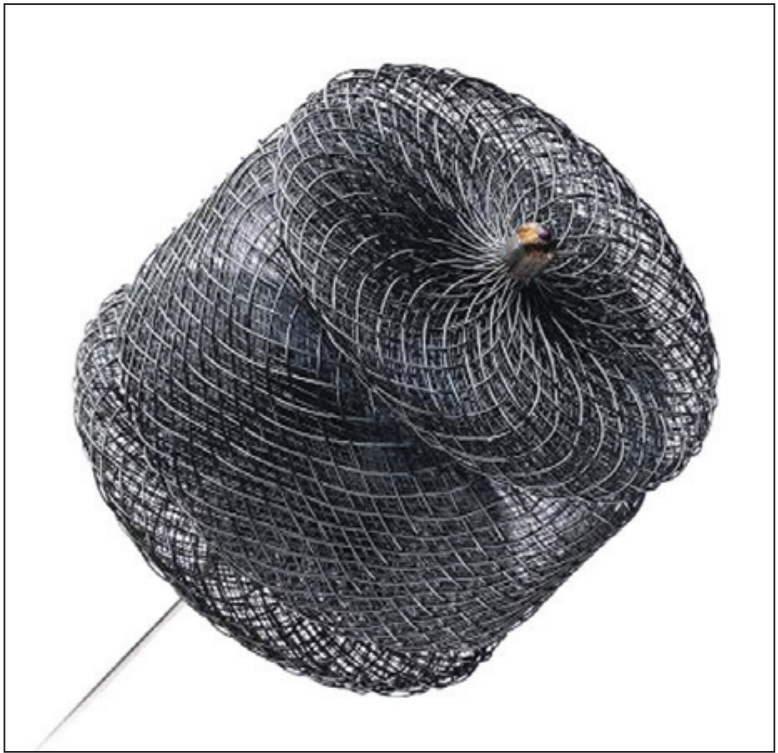

Figura 4. Dispositivo AMPLATZER ${ }^{\circledR}$ Plug Vascular II.

composta por malha de nitinol não revestida, de baixo perfil, que não requer uso de bainha e que possibilita o reposicionamento e a eventual remoção do dispositivo. O plug é compatível com cateteres-guia de 5 a $7 \mathrm{~F}$, na dependência da prótese selecionada, e tem a recomendação de ser 30 a 50\% maior que o diâmetro do vaso alvo, para minimizar o risco de embolização distal (Figura 4).

Inicialmente idealizados para embolizações periféricas, diferentes tipos de plugs têm sido utilizados com sucesso e baixa incidência de complicações no tratamento de anormalidades vasculares associadas a cardiopatias congênitas. Em 2007, Fischer et al. ${ }^{9}$ relataram três casos de oclusão percutânea de FAV congênitas utilizando esse dispositivo vascular. Mylonas et al. ${ }^{10}$ descreveram a oclusão percutânea de um aneurisma de $9 \mathrm{~cm}$ de diâmetro em enxerto de veia safena degenerado para ramo marginal obtuso em um paciente de 85 anos. Foi utilizado um plug vascular de $8 \mathrm{~mm}$, com sucesso imediato do procedimento, confirmado em controle tomográfico em 30 dias.

A embolização percutânea para o tratamento de FAV iatrogênicas pós-revascularização do miocárdio tem se mostrado método seguro e eficaz, firmando-se como o de escolha frente à correção cirúrgica. ${ }^{4}$

Apresentamos aqui uma alternativa, ainda não relatada na literatura, à clássica utilização de coils ou balões destacáveis no tratamento dessa condição específica. A embolização percutânea com oclusor vascular mostrou-se tecnicamente simples, todavia com custo ainda elevado. Seguimentos tardios são necessários para avaliar a durabilidade do efeito oclusor bem como o risco de complicações, sobretudo de embolização distal e de perfuração vascular.

\section{CONFLITO DE INTERESSES}

Não há.

\section{FONTE DE FINANCIAMENTO}

Não há.

\section{REFERÊNCIAS}

1. Calkins JB Jr, Talley JD, Kim NH. Iatrogenic aorto-coronary venous fistula as a complication of coronary artery bypass surgery: patient report and review of the literature. Cathet Cardiovasc Diag. 1996;37(1):55-9.

2. Khunnawat C, Mukerji S, Abela GS, Thakur RK. Unusual complications of coronary artery by-pass graft surgery. Am J Cardiol. 2006;98(12):1665-6.

3. McNulty $\mathrm{PH}$, Gilchrist IC. Natural history of inadvertent aorta-saphenous vein-coronary vein by-pass graft. Ann Thorac Surg. 2003;75(3):996-7.

4. Gardner JD, Maddox WR, Calkins JB Jr. Iatrogenic aortocoronary arteriovenous fistula following coronary artery by-pass surgery: a case report and complete review of the literature. Case Reports Cardiol [Internet]. 2012 [cited 2014 Jan 26]. Available from: file://C:/Documents\%20and\%20Settings/2509501/Meus\%20 documentos/Downloads/652086.pdf

5. Scholz KH, Wiegand V, Rosenmeyer P, Chemnitius, Kreuzer $\mathrm{H}$. Aorto-coronary artery to coronary vein fistula with the potential of coronary steal as complication of saphenous jump by-pass graft. Eur J Cardiothorac Surg. 1993;7(8):441-2.

6. Deligonul U, Vandormael M, Harper JM, Kern MJ. Spontaneous closure of iatrogenic left internal mammary artery to anterior interventricular vein fistula. Am J Cardiol 1986;58(9):854-5.

7. Klinke WP, Pepine CJ, Conti CR. Demonstration of an inadvertently created aorto-coronary venous anastomosis: evidence against the clinical effectiveness of retrograde coronary perfusion. Cathet Cardiovasc Diagn. 1979;5(4):367-70.

8. Lopez JJ, Kuntz RE, Baim DS, Johnson RG, Kim D. Percutaneous occlusion of an iatrogenic aortosaphenous vein-coronary fistula via retrograde coronary sinus approach. Cathet Cardiovasc Diagn. 1996;37(3):339-41

9. Fischer G, Apostolopoulou SC, Rammos S, Kiaffas M, Kramer $\mathrm{HH}$. Transcatheter closure of coronary arterial fistulas using the new Amplatzer vascular plug. Cardiol Young. 2007;17(3):283-7.

10. Mylonas I, Sakata Y, Salinger MH, Feldman T. Successful closure of a giant true saphenous vein graft aneurysm using the Amplatzer vascular plug. Catheter Cardiovasc Interv. 2006;67(4):611-6. 